\title{
The key technology research of higher vocational education cloud platform in Guizhou based on big data
}

\author{
Yuan Linlin ${ }^{1, a^{*}}$, Qin Jin ${ }^{2, b}$,Huang Ying ${ }^{3, c}$ \\ ${ }^{1}$ Guizhou Vocational Technical Institute, Guiyang 550023,Guizhou,China \\ ${ }^{2}$ Computer Science and Technology, GuiZhou University, Guiyang 550023, Guizhou,China \\ ${ }^{3}$ Guizhou Vocational Technical Institute, Guiyang 550023,Guizhou,China \\ a120362538@qq.com, 'linlinqizhi@qq.com, ‘34067445@qq.com
}

Keywords: higher vocational; education cloud platform; big data

Abstract. designed and implemented a set of typical education cloud platform system, the platform adopts the double virtual network architecture, the network is divided into two layers: the underlying real device based on virtual support platform, to build the business layer.

\section{Introduction}

At present, the education informatization application has entered the transition period, is gradually by building the campus network and other infrastructure platform to deepen the application, application to drive the development of modern education transformation development. How to make use of a new generation of information technology, to realize the rapid development of education informatization education informatization construction is facing new challenges. And with the popularity of cloud computing technology and application, and promote the development of desktop virtualization, compared with the traditional PC application solutions for its low cost, high reliability, low maintenance, high security, the characteristics of flexibility, more and more popular in the education industry[1].

On the fifth plenary session of the 17 th, the state council issued "the state council on speeding up cultivating and developing strategic emerging industries decided to $»$, put forward to develop the strategic emerging industries, the GDP ratio rose from $8 \%$ in 2015 to $15 \%$ in 2020 . A new generation of information technology as an important branch of strategic emerging industries, also got " " decision support, a reference to promote the Internet of things, cloud computing research and demonstration application.

From domestic market penetration, "twelfth five-year" period will be cloud computing industry the most critical period of growth, the cloud computing market will enter the growth stage, the industry will be rapid growth. Is expected to around 2015, cloud computing applications will begin to enter the mature stage of development. The cloud services over the next five years the average annual compound growth rate is expected to reach more than $30 \%$, the development speed is more than 6 times of traditional IT industry.

\section{BIG DATA AND CLOUD PLATFORM ADVANTAGES}

Project construction from school practical teaching requirements, higher vocational education in guizhou cloud platform can have the following advantages[2]:

1) Effectively improve the quality of teaching, change the "teacher-centered" passive learning mode, for the "student-centered" mode of active learning.

Currently the vast majority of school teaching and teachers taught in the form of PPT, not according to the specific needs of each professional course provides students with the corresponding teaching scenario. After class the students no longer the unfinished homework in class learning or teaching content.

Desktop virtualization can according to the different curriculum teaching scenarios, such as database applications need Windows XP, this course teaching VFP software need, you can 
according to the specific course requirements set specific environment configuration, formed an independent teaching scene database applications.

A teacher has account and password to login his desktop whether he is at home, in office or classroom, no need to take laptop. (see the Figure1)

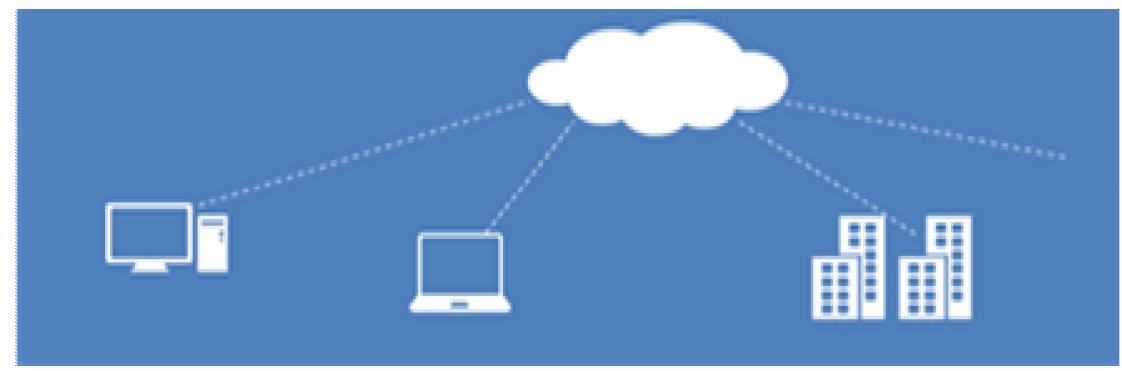

Fig .1: cloud platform advantages

According to the actual teaching situation when logging students each class can get a clean, perfect learning environment for a new desktop. Students in class can switch at any time the operating system and running environment, achieve the goal of flexible teaching. Schedule automatic import, attends class reservation function such as automatic startup for desktop virtualization and the combination of teaching, greatly reduce the work burden of administrators, improve the efficiency of teaching; Screen broadcast, lock screen, and other functions to let the teacher in class more efficient, the screen broadcast, convenient for every student can clearly see the teacher's operation and fine interpretation, lock screen function to let the students listen to the teacher only when listening to the teacher, can't surf the Internet or play games, effectively ensure the quality of multiple media teaching in the teaching.

2) Efficient unified management, reduce working intensity of management personnel

Desktop virtualization platform through the desktop computing resource pooling management, implement flexible rapid deployment, on demand. For desktop deployment and distribution, the administrator only on making image template management stage, after a batch produced and restart dozens or even hundreds of desktop, and the whole process but over ten minutes. After pooling resources management, can open desktop resources in resource utilization is low for students with special needs, time and billing, do according to the need to use, pay by using, make full use of IT resources.

Desktop virtualization as a way of cloud computing, the requirement to the terminal equipment is greatly reduced, can use a variety of computing terminal access to use desktop virtualization platform for virtual desktop. Desktop virtualization platform based on server computing architecture greatly reduce the computing needs of front-end equipment, so as to prolong the service life of the original PC terminal, with the elimination of old equipment can keep monitor continue to use, save large amount of desktop terminal input costs[3].

\section{THE KEY TECHNOLOGIES OF PLATFORM DESIGN}

1) Big data search algorithm

Once the data has been selected, the next task is to determine which of the implemented learning methods to apply. Each algorithm applies different search strategies and is applicable on only specific types of data. Beyond data selection, knowing which algorithm to use is significant challenge to making effective use of a data mining tool. In the following sections, each algorithm will be explained in terms of the data upon which it acts and examples are given for when it is applicable.

The selection of a search algorithm depends on the characteristics of the database. Applying different algorithms to the same database will lead to different discovered knowledge. A search approach can be either bottom-up or top-down. Bottom-up search uses a sequence of generalization operations to generalize the concepts; however, a top-down approach modifies the initial description by both generalization and specialization operations until the rules classify the training set correctly. 
The size of the training set determines the search strategy. The simplest strategy is an exhaustive search which applies to the entire set. However, in a real application, the search space is often large enough to make an exhaustive search inefficient. Therefore, some modified strategies were developed for improving the search efficiency. If the search strategy is represented as a tree, then the nodes are the set of objects and the edges represent operations. The initial structure is the root of the tree. The system navigates, by operations, through the search space. If there is only one operation with the best quality applied, it is a hill climber; if there are $\mathrm{n}$ best operations selected, it is a beam search where $\mathrm{n}$ is the beam width; or if all operations are fully developed, then it is called an exhaustive search. The relation among these three can be represented in the Figure 2

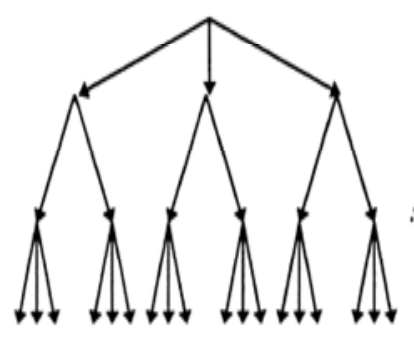

Exhaustive search

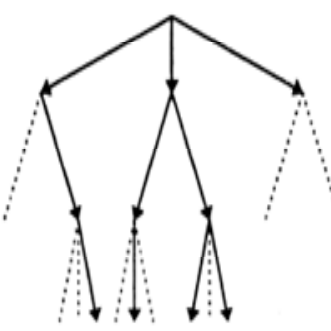

Beam search

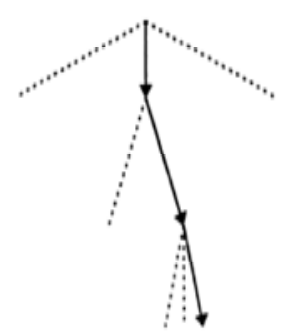

Hill climber

Fig. 2: Search strategies

Classification-rule learning involves finding rules or decision trees that partition given data into predefined classes. For any realistic problem domain of the classification-rule learning, the set of possible decision trees is too large to be searched exhaustively. In fact, the computational complexity of finding an optimal classification decision tree is NP hard. Most of the existing induction-based algorithms use Hunt's method as the basic algorithm. Here is a recursive description of Hunt's method for constructing a decision tree from a set $\mathrm{T}$ of training cases with classes denoted $\{\mathrm{C} 1, \mathrm{C} 2, \ldots, \mathrm{Ck}\}$.

Case $1 \mathrm{~T}$ contains one or more cases, all belonging to a single class $\mathrm{Cj}$ : The decision tree for $\mathrm{T}$ is a leaf identifying class $\mathrm{Cj}$.

Case $2 \mathrm{~T}$ contains no cases: The decision tree for $\mathrm{T}$ is a leaf, but the class to be associated with the leaf must be determined from information other than $\mathrm{T}$.

Case $3 \mathrm{~T}$ contains cases that belong to a mixture of classes: A test is chosen, based on a single attribute, that has one or more mutually exclusive outcomes $\{\mathrm{O} 1, \mathrm{O} 2, . ., \mathrm{On}\}$. $\mathrm{T}$ is partitioned into subsets $\mathrm{T} 1, \mathrm{~T} 2, \ldots, \mathrm{Tn}$, where Ti contains all the cases in $\mathrm{T}$ that have outcome Oi of the chosen test. The decision tree for $\mathrm{T}$ consists of a decision node identifying the test, and one branch for each possible outcome. The same tree building machinery is applied recursively to each subset of training cases.

2) education cloud platform stucture

Virtualization platform is a distributed computing platform, overall architecture as shown(see the Figure3): 


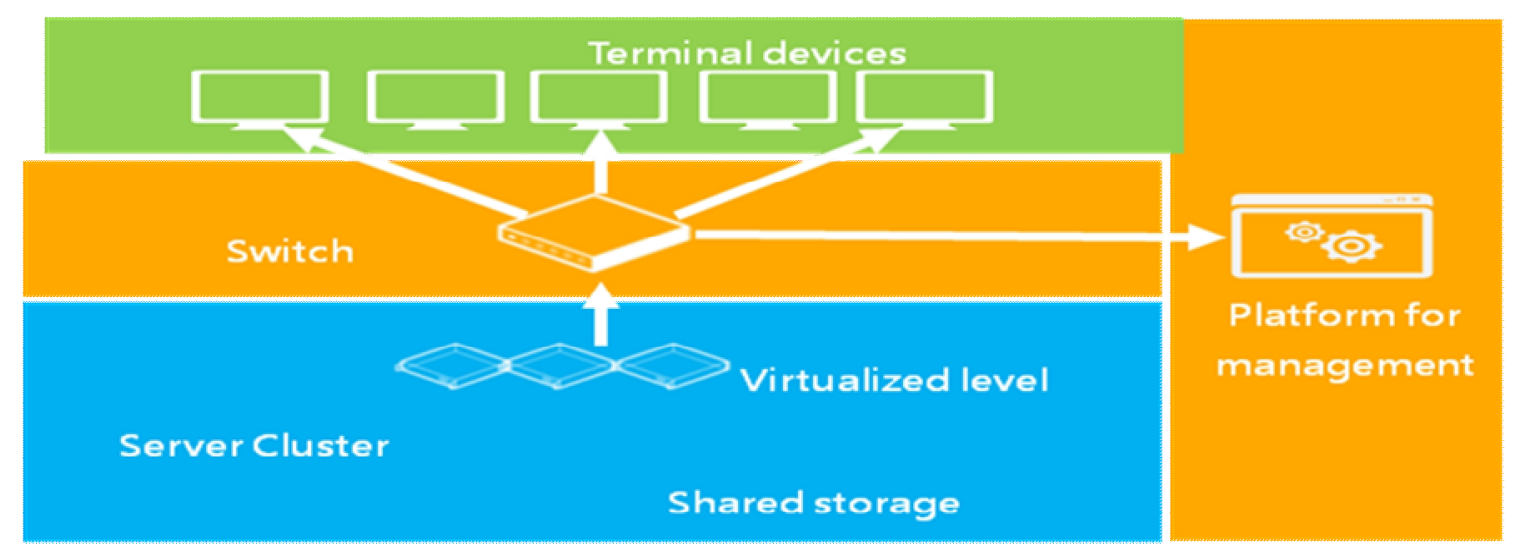

Fig.3: cloud platform stucture

Cloud computing is the future development trend of education informatization platform, while education cloud with special requirements according to their aptitude, universal cloud platform is not very suitable. In view of the personalized needs in the field of education, the author participated in the team's education cloud platform design and implementation. Platform system adopts double virtual network architecture, in terms of scalability, flexibility, safety, high efficiency advantages, have education cloud need all kinds of big data processing capabilities.

\section{SUMMARY}

Education cloud platform research and development is a long-term work, there are still a lot of future application research and development as well as the core technology research and development work to do. Overall architecture design, the cloud platform has been done of virtualization, cloud computing resources and resource overdraw function, the future will also further deepen resources use way and architecture, trying to maximize resource utilization, using the risk minimization. Can draw lessons from the financial credit in the field of risk assessment and control model, the cloud computing platform to establish similar financial resources reserve mechanism of the deposit reserve system.

\section{Acknowledgments}

This paper is supported by the Commissioned by the national Open University, number :G14A3602W; This paper is supported by the Guizhou institute of vocational and technical college vocational education subject, number : 15GH04; and The National Natural Science Fund;NO:61562009, and The National Natural Science Fund;NO:61562009; and the Guizhou University to introduce talents research project NO:[2012]028

\section{References}

[1] Jian-cheng QIN,Zhong-ying BAI. Design of new format for mass data compression[J]. The Journal of China Universities of Posts and Telecommunications . 2011 (1)

[2] Michael Armbrust,Armando Fox,Rean Griffith,Anthony D. Joseph,Randy Katz,Andy Konwinski,Gunho Lee,David Patterson,Ariel Rabkin,Ion Stoica,Matei Zaharia. A view of cloud computing[J]. Communications of the ACM . 2010 (4)

[3] Min Long,Fei Peng,Shuisheng Qiu,Yanfeng Chen. Implementation of a new chaotic encryption system and synchronization 11 This project was supported by the National Natural Science Foundation of China (60372004), and the Natural Science Foundation of Guangdong Province (31445, 20820).[J]. Journal of Systems Engineering and Electronics . 2006 (1) 